\title{
Stress responsivity in children with externalizing behavior disorders
}

\author{
HEDDEKE SNOEK,${ }^{a}$ STEPHANIE H.M. VAN GOOZEN, ${ }^{b}$ \\ WALTER MATTHYS, ${ }^{a}$ JAN K. BUITELAAR ${ }^{c}$, AND \\ HERMAN VAN ENGELAND ${ }^{a}$ \\ ${ }^{a}$ University Medical Center Utrecht, Rudolf Magnus Institute for Neurosciences; \\ ${ }^{b}$ University of Cambridge; and ${ }^{c}$ University Medical Center Nijmegen
}

\begin{abstract}
Patterns of lower autonomic nervous system (ANS) and hypothalamic-pituitary-adrenal (HPA) axis activity have been found in children with oppositional defiant disorder (ODD). The aim of the present study was to investigate whether children with attention-deficit/hyperactivity disorder (ADHD) differ from ODD children with (OD/AD) or without comorbid ADHD in ANS and HPA axis activity under baseline and stressful conditions. The effects of stress on cortisol, heart rate (HR), and skin conductance level (SCL) were studied in 95 children (26 normal control [NC] children and 69 child psychiatric patients referred for externalizing behavior problems [15 ODD, 31 OD/AD, and $23 \mathrm{ADHD}]$ ). No baseline differences were found in cortisol between the four groups. However, the ODD and $\mathrm{OD} / \mathrm{AD}$ groups showed a significantly weaker cortisol response to stress compared to the ADHD and NC groups; the ADHD group had a similar cortisol response as the NC group. Within the ODD group this pattern of low cortisol responsivity was most clearly present in the more severely affected inpatients. With respect to HR, the ODD group had a significantly lower HR during baseline and stressful conditions. The higher HR levels in the OD/AD and ADHD groups were likely to be caused by methylphenidate. The externalizing groups had significantly lower SCL levels, and no differences were found between these groups. It was concluded that differences in cortisol responsivity during stress exposure are important in distinguishing within a group of children with externalizing behavior between those with ODD and ADHD.
\end{abstract}

Children who show persistent noncompliant antisocial and aggressive behavior receive a diagnosis of conduct disorder (CD; Diagnostic and Statistical Manual of Mental Disorders4th ed. [DSM-IV]; American Psychiatric Association [APA], 1994) or oppositional defiant disorder (ODD). Studies on the biology of these disorders in young children are important, because antisocial and criminal adults often have their onset of deviant behavior in childhood (Loeber \& Stouthamer-Loeber, 1998). There is increasing evidence that aggressive children, and especially those who show per-

Address correspondence and reprint requests to: Heddeke Snoek, Department of Child and Adolescent Psychiatry, A01.468, University Medical Center Utrecht, P.O. Box 85500, 3508 GA Utrecht, The Netherlands; E-mail: h.snoek@psych.azu.nl. sistent antisocial behavior over the years, are characterized by neurobiological problems. For example, stress-regulating mechanisms, including the hypothalamic-pituitary-adrenal (HPA) axis and the psychophysiological autonomic nervous system (ANS) have been found to be important in explaining individual differences in antisociality (Kruesi, Hibbs, Zahn, Keysor, Hamburger, Bartko, \& Rapoport, 1992; Raine, Venables, \& Williams, 1990; Vanyukov, Moss, Plail, Blackson, Mezzich, \& Tarter, 1993). One influential biological theory of antisocial behavior is that antisocial individuals are underaroused. Two theoretical interpretations have been offered to explain patterns of reduced arousal in antisocials. The fearlessness theory claims that low levels of arousal are markers of low levels of fear (Raine, 1993). According to this theory, one can hypothesize 
that fearless children are more likely to engage in physical fights to obtain rewards and social status because they do not fear the negative consequences of their aggressive actions due to a lack of fear conditioning. A different explanation is provided by the stimulation-seeking theory (Zuckerman, 1979), which argues that low arousal represents an aversive physiological state and that individuals with tonically low arousal are motivated to seek out stimulation in order to raise their arousal levels to an optimal or normal level.

Several investigations of antisocial adults have analyzed cortisol levels and observed a negative relationship with the magnitude of behavioral deviation (Virkkunen, 1985; Woodman, Hinton, \& O'Neill, 1978). Few studies have been conducted on cortisol in children, and the findings are equivocal. Some studies have found the expected inverse associations between cortisol and aggression (Kariyawasam, Zaw, \& Handley, 2002; McBurnett, Lahey, Rathouz, \& Loeber, 2000; Pajer, Gardner, Rubin, Perel, \& Neal, 2001; Vanyukov et al., 1993); other studies found no relationship (Kruesi, Schmidt, Donnelly, Hibbs, \& Hamburger, 1989; Scarpa \& Kolko, 1994; Schulz, Halperin, Newcorn, Sharma, \& Gabriel, 1997; Stoff, Pasatiempo, Yeung, Cooper, Bridger, \& Rabinovich, 1992). Some studies also found associations between reduced basal cortisol concentrations and aggression to peers (Tennes, Kreye, Avitable, \& Wells, 1986) or hostility to teachers (Tennes \& Kreye, 1985) in a normal healthy population. On the other hand, Klimes-Dougan, Hastings, Granger, Usher, and Zahn-Waxler (2001) found no evidence of a relationship between externalizing problems and reduced activity of the HPA axis in adolescents at risk for psychopathology. McBurnett, Lahey, Frick, Risch, Loeber, Hart, Christ, and Hanson (1991) found that anxious CD children had higher cortisol levels than children with CD alone. Part of the explanation for these mixed results could be that the existing studies have used different methods for collecting cortisol (salivary vs. plasma, basal state vs. stress reaction), that they defined aggression in different ways, that they used normal children or clinical cases as participants, and that they involved single measure- ments of cortisol under varying conditions. Only a few studies have measured cortisol repeatedly. Moss, Vanyukov, and Martin (1995) found that sons of fathers with a psychoactive substance use disorder secreted less cortisol in anticipation of stress. Van Goozen, Matthys, Cohen-Kettenis, Gispen-de Wied, Wiegant, and Van Engeland (1998) and Van Goozen, Matthys, Cohen-Kettenis, Buitelaar, and Van Engeland (2000) found that ODD children had lower cortisol levels when exposed to frustration and provocation than normal control (NC) participants. Specifically, the latter study found that ODD children and NC children did not differ during baseline procedures, but that the predicted stress-induced increase in saliva cortisol in the $\mathrm{NC}$ was absent in the ODD group. In the present context it is important to realize that studies using normal healthy participants or community samples have found that engaging in aggressive behavior results in an increase in cortisol level (Gerra, Zaimovic, Avanzini, Chittolini, Giucastro, Caccavari, Palladino, Maestri, Monica, Delsignore, \& Brambilla, 1997; Scarpa, Fikretoglu, $\&$ Luscher, 2000). These results suggest that a pattern of low cortisol reactivity during stress could indeed be a specific characteristic of aggressive patients (CD/ODD) and is not related to the display of aggressive behavior per se.

With respect to the ANS, there are a number of studies indicating that antisocial individuals are characterized by reduced activity in the electrodermal domain and heart rate (HR; Raine, 1996). Raine et al. (1990) found lower ANS activity in adolescents who were later sentenced for crime. In a follow-up study, Kruesi et al. (1992) found that skin conductance (SCL) significantly negatively correlated with later institutionalization. However, research findings have also been conflicting (Fowles \& Furuseth, 1994; Raine \& Venables, 1984). The relatively few studies that have been carried out with CD children indicate lower levels of ANS activity (see a review by Lahey, McBurnett, Loeber, \& Hart, 1995). Iaboni, Douglas, and Ditto (1997) found also lower levels of ANS activity in children with attention-deficit/hyperactivity disorder (ADHD) with or without CD/ODD. However, 
Zahn and Kruesi (1993) found no evidence of lower ANS baselines in boys with CD, ODD, and/or ADHD, although ANS reactivity was lower. Although the earlier mentioned Van Goozen et al. studies (1998; Van Goozen, Matthys, et al., 2000) found that children with ODD had lower baseline HR and SCL levels, there were conflicting results with respect to HR responsivity during stress: in the first study, HR levels were higher in the ODD group during provocation and frustration compared to the normal controls, but this interaction was absent in the latter study. Results of a meta-analysis by Raine, Venables, and Mednick (1997) indicate that low resting HR is the best-replicated biological marker of antisocial and aggressive behavior in childhood and adolescent community samples. Raine et al. (1997) even found some support for a psychiatric specificity of the low-HR aggression relationship in that it was independent of the presence or absence of comorbid hyperactivity.

In addition to these possible explanations for existence of conflicting results, the heterogeneity of the samples could also have been an influencing factor. The samples of the previously mentioned clinical studies included children with disruptive behavior (i.e., CD or ODD) and children with (comorbid) ADHD. These diagnoses often co-occur in one individual (the reported prevalence rates of comorbid ADHD and CD/ODD range between approximately 65 and 90\%), and both ADHD and $\mathrm{CD} / \mathrm{ODD}$ children have been found to be at risk for antisocial behavior in adolescence and adulthood (Frick \& Loney, 1999; Mannuzza \& Klein, 1999). However, it has also been suggested that antisocial behavior found in the long-term outcome of ADHD may be a function of the CD comorbidity in these children (Mannuzza \& Klein, 1999). At present, it is therefore not clear whether a pattern of lower ANS and HPA activity is generally present in the whole category of externalizing behavior disorder or is only more specifically present in children with CD/ODD. If we were to find that lower ANS and HPA axis reactivity is present in children with ODD/CD but not in cases with ADHD alone, we could have a biological factor that explains resistance to treatment in children with aggressive and antisocial behavior. These findings should ultimately result in earlier and more effective interventions for aggressive children.

Within the psychological literature, there is substantial evidence to claim that ADHD and CD/ODD are independent disorders (Schachar, 1991) with differential correlates; for example, it has been suggested that ADHD is related to cognitive deficits (Pennington \& Ozonoff, 1996) and that CD has more to do with psychosocial factors such as dysfunctional family systems and low family income (Abikoff \& Klein, 1992; Hinshaw, 1992; Schachar \& Logan, 1990). In addition, within the neurobiological domain a few studies have actively focused on differences between CD/ODD and ADHD children. For example, Halperin, Vanshdeep, Siever, Schwartz, Matier, Wornell, and Newcorn (1994), using a pharmacochallenge paradigm, found indications of a more responsive serotonergic system in aggressive boys with ADHD than in nonaggressive ADHD boys. Van Goozen, Van den Ban, Matthys, Cohen-Kettenis, Thijssen, and Van Engeland (2000) were able to make a qualitative distinction between ADHD and ODD children based on plasma dehydroepiandrosterone sulfate (DHEAS) levels, with ODD children showing the highest DHEAS levels. Finally, Herpertz, Wenning, Mueller, Qunaibi, Sass and Herpertz-Dahlmann (2001) found low autonomic responses to orienting and aversive startling stimuli in children with ADHD and CD but not in children with ADHD alone. On the other hand, in their studies on HPA axis and ANS activity Van Goozen et al. (1998; Van Goozen, Matthys, et al., 2000) found no differences in cortisol, HR or SCL between ODD children with or without comorbid ADHD. However, this study did not include ADHD children without ODD.

The aim of the present study was to investigate whether ADHD children differ from ODD children with (OD/AD) or without comorbid ADHD (ODD) in ANS and HPA axis activity under baseline and stressful conditions. As mentioned already, low arousal has been linked to fearlessness and stimulationseeking behavior, and may ultimately predispose an individual to aggressive and antisocial behavior (Raine, 1996). Therefore, we pre- 
dicted ODD and OD/AD children to have decreased HR and SCL levels and to be more aggressive than ADHD children. In addition, we expected to find lower cortisol levels in ODD and OD/AD children than in ADHD children, and that such a pattern was particularly evident when the participants were psychologically challenged. Data from a normal control group were added to the design of the study in order to be able to conclude that ADHD children indeed have normal levels of SCL, HR, and cortisol.

\section{Method}

\section{Subjects}

Participants $(n=70: 15$ ODD children, 32 OD/AD children, and 23 ADHD children) were all Caucasian and aged between 7 and 12 years (mean age ODD group $=10.4, S D=0.9$; mean age $\mathrm{OD} / \mathrm{AD}$ group $=10.0, S D=1.6$; mean age $\mathrm{ADHD}$ group $=9.8, S D=1.4 ; F(2,67)=$ $1.06, p=.35)$. All subjects were solicited from the Department of Child and Adolescent Psychiatry, University Medical Center, Utrecht, The Netherlands. Seventy children participated, but one child (an OD/AD subject) had to be excluded later because of neuroleptic medication. The ODD group $(n=46)$ consisted of boys $(n=29)$ and girls $(n=7)$ who met the criteria for ODD $(n=34)$ or $\mathrm{CD}(n=$ 12 ) as set out in the DSM-IV (APA, 1994). Because in school-aged children ODD and CD are highly interrelated (Lahey, Loeber, Quay, Frick, \& Grimm, 1992), no distinction was made between subjects who fulfill the criteria for one or both of these categories. Within the total ODD group, 27 subjects received a $24-\mathrm{hr}$ treatment in an inpatient clinic specialized in treatment of children with ODD because of the severity of their pathology, and 19 subjects received an intensive " 8 a.m. to 4 p.m." day treatment. The ADHD subgroup (19 boys and 4 girls) met the DSM-IV diagnosis of ADHD. The OD/AD subgroup met the $D S M-I V$ criteria for ODD and ADHD. Diagnoses of the subjects were based on psychological assessment and psychiatric interviews with the child, interviews with the parents, including discussion of the child's developmental history and observations by child care workers), and the administration of an extensive semistructured interview (Diagnostic Interview Schedule for Children version 2.3 [DISC-P]; Fisher, Wicks, Shaffer, Piacentini, \& Lapkin, 1992) in which all possible DSM-IV diagnosis were systematically explored. A trained graduate educational psychologist administered this DISC-P. On the basis of information from these various informants, consensus on the diagnosis was reached between three psychiatrists (W.M., J.K.B., H.v.E.).

In order to be able to conclude that ADHD children have a more normal pattern of ANS and HPA axis activity during stress, we included the published data of a group of normal healthy control children $(n=26)$, which were collected as part of an earlier study in which the same procedure was used (Van Goozen, Matthys, et al., 2000). These normal control (NC) children were recruited from Grades 3-6 of regular elementary schools (mean age $=10.3$ years, $S D=1.3)$. None of the NC children were using any (psycho) pharmacological medication.

Exclusion criteria for all participants were any neurological disorder and IQ less than 75 . The psychopharmacological medication used by the subjects has been listed in Table 1. For clinical reasons it was decided not to stop the medication. From Table 1 it becomes clear that a large proportion of the clinical cases was on MPH $(n=36)$. However, although MPH has a mildly stimulating effect on the cardiovascular system, the evidence also indicates that chronic oral MPH treatment has no effect on plasma cortisol levels (Weizman, Dick, GilAd, Weitz, Tyano, \& Laron, 1987).

To describe participants from a dimensional point of view the Child Behavior Checklist (CBCL; Achenbach, 1991) was completed by the parents of all subjects (see Table 1 for a detailed description of the demographic and clinical characteristics of the diagnostic groups).

The study was approved by the Medical Ethical Committee of University Medical Center Utrecht. Written informed consent was obtained from the parents and verbal assent was obtained from the child. 
Table 1. Demographic and clinical characteristics of the diagnostic groups

\begin{tabular}{|c|c|c|c|c|c|}
\hline Diagnostic Groups & NC & ODD & $\mathrm{OD} / \mathrm{AD}$ & ADHD & $F$ Value \\
\hline Number of subjects & 26 & 15 & 31 & 23 & - \\
\hline Boys/girls ratio & $20 / 6$ & $12 / 3$ & $27 / 4$ & $19 / 4$ & - \\
\hline Medication use (number of patients) & - & $\begin{array}{l}\text { Fluvoxamine }+ \\
\quad \text { risperidone (1) }\end{array}$ & $\begin{array}{l}\text { MPH (17), MPH + } \\
\quad \text { clonidine (1), MPH + } \\
\text { risperidone (1), } \\
\text { desipramine (1), } \\
\text { clonidine (1) }\end{array}$ & MPH (17) & - \\
\hline Number of CD cases & - & 1 & 11 & - & - \\
\hline Number of inpatients & - & 11 & 16 & - & - \\
\hline Comorbid diagnosis according to DISC-P & - & $\begin{array}{l}\text { Depression (3), } \\
\text { mania (1), } \\
\text { anxiety disorder (9), } \\
\text { Tourette (1), } \\
\text { enuresis (2), } \\
\text { encopresis (1) }\end{array}$ & $\begin{array}{l}\text { Depression (4), } \\
\text { anxiety disorder (9), } \\
\text { Tourette/tic disorder (2), } \\
\text { enuresis (2), } \\
\text { encopresis (1) }\end{array}$ & $\begin{array}{l}\text { Anxiety disorder (13), } \\
\text { Tourette/tic disorder (5), } \\
\text { enuresis (1), } \\
\text { encopresis (2) }\end{array}$ & - \\
\hline \multicolumn{6}{|l|}{ CBCL } \\
\hline Delinquent behavior & $51.5^{a}$ & $71.8^{b}$ & $73.4^{b}$ & $56.0^{a}$ & 68.0 \\
\hline Aggressive behavior & $52.0^{a}$ & $79.7^{b}$ & $82.8^{b}$ & $61.5^{c}$ & 78.7 \\
\hline Externalizing behavior & $44.3^{c}$ & $76.9^{b}$ & $78.8^{b}$ & $60.1^{c}$ & 98.6 \\
\hline
\end{tabular}

Note: NC, normal controls; ODD, oppositional defiant disorder; OD/AD, oppositional defiant disorder with comorbid ADHD; ADHD, attention-deficit/hyperactivity disorder; MPH, methylphenidate. ${ }^{a}$ Differed significantly from ODD and OD/AD groups.

${ }^{b}$ Differed significantly from NC and ADHD group.

${ }^{c}$ Differed significantly from all other three groups. 


\section{Procedure}

Prestress phase (PRE). The subjects spent 30 min filling out questionnaires and watching film clips.

Stress phase (STRESS). Stress was induced for $80 \mathrm{~min}$ and involved frustration, provocation, and aggression in a general setting of competition between the real participant and a videotaped opponent of similar age and sex, who competed with the subject for best performance (Van Goozen et al., 1998; Van Goozen, Matthys, et al., 2000). In the first block (3) and the last block (7) of the STRESS phase the participants were administered a response perseveration task in which they could win or lose money (for details, see Daugherty \& Quay, 1991). Next, frustration was induced by having the subject perform a difficult computer task under time pressure while the video opponent was watching. In this task the children had to move their cursor quickly and precisely to specific targets on their screen. After every trial they received feedback and were usually told to be faster and/or more precise. In this way, all participants were made to perform badly and were told by the video opponent on completion of this test to do it once more. The second time the task was programmed in such a way that it was even more frustrating. An opportunity for aggression was provided to the participant after s/he had been provoked twice by the competitor. This provocation was standardized by using the videotape of the competitor, who criticized the performance of the participant in a competitive and derogatory way. Next the video opponent had to perform a difficult task and the participant could give him/her feedback by pressing buttons containing a reward signal or white noise, the intensity level of which could be varied. The aggression intensity was registered. None of the participants was aware that the video opponent was not a real participant.

Poststress phase (POST). For 30 min: after the stress phase the participant filled in questionnaires, watched videos, and was told $\mathrm{s} / \mathrm{he}$ was the winner of the competition.

\section{Procedure for saliva cortisol collection and analysis}

Each subject participated in the experiment between 1 and 4 PM because afternoon values are more strongly influenced by external stimulation (Kirschbaum, Steyer, Eid, Patalla, Schwenkmezger, \& Hellhammer, 1990). Seven saliva samples were collected (cort1-cort7). The last two samples were taken within $30 \mathrm{~min}$ after the end of the stress part, and no proper poststress sample was therefore collected. The implication of this, and the fact that it takes relatively long (about 20-30 $\mathrm{min}$ ) to induce an increment in cortisol (Kirschbaum \& Hellhammer, 1989) is that the first two cortisol samples constitute the PRE samples and the last five samples the STRESS phase samples (Van Goozen, Matthys, et al., 2000).

Saliva samples were collected in plastic vials after saliva production was stimulated with citric acid (not more than two crystals at each sampling) and were stored at $-20^{\circ} \mathrm{C}$ until analysis.

Cortisol concentrations were measured without extraction using an in-house competitive radioimmunoassay with a polyclonal anticortisol antibody (K7348). [1,2- $\left.{ }^{3} \mathrm{H}(N)\right]$ Hydrocortisone (NET 185, NEN DuPont, Dreiech, Germany) was used as a tracer after chromatographic verification of its purity. The lower limit of detection was $0.5 \mathrm{nmol} / 1$ and interassay variation was $11.0,8.2$, and $7.6 \%$ at 4.7, 9.7, and $14.0 \mathrm{nmol} / 1$, respectively $(n=$ 20). Saliva cortisol levels correlate highly with serum cortisol concentrations (correlation coefficients of $r \geq .90)$ and reflect the unbound fraction of circulation cortisol (Kirschbaum \& Hellhammer, 1989).

\section{Procedure for SCL and HR registration}

Subjects were seated throughout the session and asked to remain still. Electrodermal activity (SC4 skin conductance; Contact Precision Instruments, UK) was recorded by a constant voltage $(0.5 \mathrm{~V})$ method from the distal phalanges of the middle and ring fingers of the nondominant hand using $\mathrm{Ag} / \mathrm{AgCl}$ electrodes (the contact diameter of the electrodes was $0.4 \mathrm{~cm}$ ) with electrode collars of $0.5 \mathrm{~cm}$ in 
diameter, which were filled with Hewlett Packard Redux creme. Before each recording session the skin conductance coupler was calibrated to map activity from 0 to approximately 52 microSiemens $(\mu \mathrm{S})$. A finger pulse amplifier (photoplethysmoGRAPH;1625 Contact Precision Instruments, UK) for the recording of HR was fixed to the index finger of the nondominant hand. SCL and HR were recorded nine times during the different phases. The first two samples constitute the PRE phase, the next five samples the STRESS phase, and the last two samples the POST phase.

\section{Recording of psychological states}

At nine times subjects rated their feelings. An adaptation for children of a clinical self-rating scale was used (Von Zerssen, 1986). A negative feeling score per event was calculated by adding scores of eight dichotomous items ("I feel: happy-gloomy, well-sick, cheerful-not cheerful, good-bad, loved-not loved, satisfieddissatisfied, afraid-not afraid, strong-weak"). The first two rating were done in the PRE phase, the next five samples represent the STRESS phase, and the last two ratings were done after they had been told the outcome of the competition.

\section{Data analysis}

Repeated-measures multivariate analyses of variance (MANOVAs) with "group" (NC vs. ODD vs. OD/AD vs. ADHD) as betweensubjects factor and "time" as within-subjects factor were used to assess changes in levels of cortisol, SCL and HR and negative mood as a result of different manipulations. HuyhnFeldt corrections were used where the assumption of sphericity was violated. Main effects of "time" and interactions between "time" and "group" were further analyzed by conducting contrast tests. In the event of significant interactions, one-factor ANOVAs were used to assess the effect of diagnostic group on the different samples, and in case of significant group differences post hoc Bonferroni tests were done. Furthermore, one-factor ANOVAs were used to examine whether there were group differences in HR, SCL, and cortisol change scores, with a change score $(\Delta)$ defined as the mean STRESS phase values minus the mean PRE phase value.

Furthermore, repeated-measures MANOVAs with different between-subjects factors (ODD or not; ADHD or not; MPH or not) and time as within-subjects factor were used to assess the independent factor effect on cortisol, HR, and skin conductance.

Single isolated missing values or single isolated outlier values, with an outlier defined as an individual value more than $2.5 S D$ s above or below the mean value of the group, were replaced by the group averages. Values are expressed as mean $(S D)$.

\section{Results}

\section{HPA axis: Cortisol}

Two subjects (1 OD/AD and 1 ADHD) were excluded from the cortisol analyses because more than four of the seven cortisol (cort) samples had outlier values. There were no significant differences in cortisol level in the PRE phase: cort1: $\mathrm{NC}=6.9 \mathrm{nmol} / 1(S D=2.6)$, $\mathrm{ODD}=6.4 \mathrm{nmol} / 1(S D=2.1), \mathrm{OD} / \mathrm{AD}=7.1$ $\mathrm{nmol} / 1(S D=1.7), \mathrm{ADHD}=5.8(S D=2.5)$ $\mathrm{nmol} / 1 ; F(3,89)=1.71, n s ;$ and cort $2: \mathrm{NC}=$ $5.8 \mathrm{nmol} / 1(S D=2.1)$, ODD $=5.4 \mathrm{nmol} / 1$ $(S D=2.3), \mathrm{OD} / \mathrm{AD}=5.8 \mathrm{nmol} / \mathrm{l}(S D=1.3)$, $\mathrm{ADHD}=6.0 \mathrm{nmol} / 1(S D=3.1) ; F(3,89)=$ $0.23, n s$. A repeated-measures MANOVA over all seven cortisol samples revealed a main effect of time, $F(6,84)=10.07, p<.001$, a main effect of group, $F(3,89)=3.09, p=$ .03 , and an interaction between group and time, $F(18,248)=2.97, p<.001$ (see Figure 1$)$.

Additional contrast tests were carried out to analyze the main effects of time and the significant interaction further. These showed that the main effect of time was primarily attributable to a general decline in cortisol between cort 1 and cort $2, F=62.44, p<.001$. The significant interaction was attributable to a decline in cortisol between cort 1 and cort 2 in the NC, ODD, and OD/AD groups, whereas there was no decline in the ADHD group, $F=$ $11.73, p<.001$, and, even more importantly, to a tendency for cortisol to continue to decline in both the ODD and OD/AD groups between 


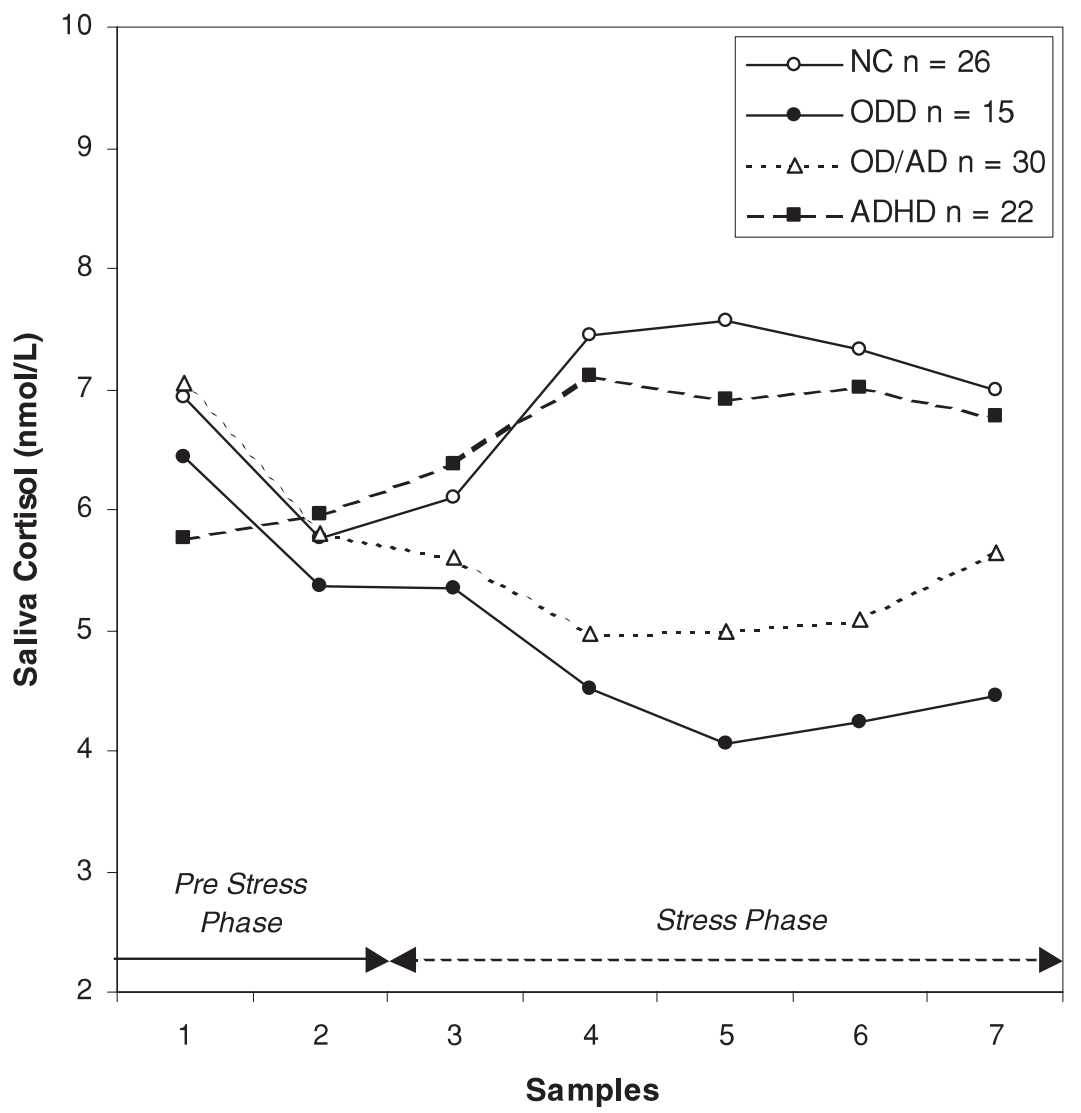

Figure 1. Mean saliva cortisol levels in normal control (NC) children and children with oppositional defiant disorder (ODD), attention-deficit/hyperactivity disorder (ADHD), and ODD with comorbid ADHD (OD/AD).

cort3 and cort4, whereas the cortisol level in both the NC and the ADHD groups increased between these samples, $F=4.25, p<.01$. Post hoc Bonferroni tests showed significant differences between the NC and OD/AD group on cort4 $(p<.05)$, between the NC group and both the ODD and the OD/AD groups on cort5 $(p<.009$ and $p<.03$, respectively), and between the NC and ODD groups on cort6 $(p<.03)$. Furthermore, when calculating change scores the ADHD group $(\Delta$ cort $=0.98$ nmol/1) differed significantly from both the ODD $(\Delta$ cort $=-1.37)$ and the OD/AD $(\Delta$ cort $=-1.17)$ groups (mean differences $=$ $2.35, p<.04$, and $2.15, p<.02$ ), whereas the ADHD group did not differ significantly from the $\mathrm{NC}$ group $(\Delta$ cort $=0.73 \mathrm{nmol} / \mathrm{l}$; mean difference score $=0.25, n s$ ).

Because 19 ADHD subjects were on MPH, we conducted a repeated-measures MANOVA with $O D D, A D H D$, and $M P H$ as separate between-subjects factors to assess their independent effect in the different cortisol response patterns. These analyses showed that only the ODD factor had a significant interaction with time, $F(6,82)=4.22, p<.002$, and that there was no interaction between time and MPH, $F$ $(6,82)=0.98, n s$, or time and ADHD, $F(6$, $82)=1.68$, ns. Moreover, these tests only showed a main effect for ODD, $F(1,87)=$ $6.44, p<0.02$. To assess the effect of MPH on cortisol reactivity, we analyzed the cortisol levels within both ODD groups (ODD and OD/AD) and compared ODD children with $(\mathrm{MPH}+; n=18)$ and without MPH (MPH-; $n=27$ ). A repeated-measures MANOVA over all 7 cortisol samples revealed main effects of time, $F(6,38)=26.02, p<.001$, and group, $F(1,43)=5.76, p=.02$, but no interaction between group and time, $F(6,38)=1.17, n s$. 
The significant group effect was due to generally higher cortisol levels in the MPH+ group: PRE phase (=mean cort $1-\operatorname{cort} 2)$ : $\mathrm{MPH}+=7.0 \mathrm{nmol} / 1, \mathrm{MPH}-=5.7 \mathrm{nmol} / 1$, $F(1,43)=6.70, p=.013$; STRESS phase $(=$ mean cort $3-$ cort 7$): \mathrm{MPH}+=5.7$, MPH $-=4.9, F(1,43)=4.02, p=.05$. There was no difference in cortisol change score levels $(\Delta$ cort $)$ and both ODD groups showed a comparable decrease in cortisol $(\Delta$ cort: $\mathrm{MPH}+=-1.4, \mathrm{MPH}-=-1.2, \mathrm{~F}$ $(1,43)=0.14, p=.71$, during the stress phase of the experimental procedure. Clearly, these findings are in marked contrast with the findings in the ADHD and NC groups, because the latter two groups showed positive cortisol change scores $(\Delta$ cort ADHD $=$ $0.98 ; \Delta$ cort $\mathrm{NC}=0.73$ ).

In a next analysis the effect of severity of the ODD pathology was assessed by comparing the cortisol response of the ODD inpatients to that of the ODD children who received day treatment. A repeated-measures MANOVA with treatment (inpatient vs. day treatment) as between-subjects factor and time as within-subjects factor showed a significant interaction (after Huyhn-Feldt adjustment) between treatment and time, $F(2$, $86.2)=5.80, p<.005$. It turned out that the pattern of low cortisol reactivity during stress was most clearly present in the more serious inpatient group.

Finally, because anxiety plays an important moderating role in cortisol response, we analyzed whether there were differences between externalizing subjects with and without an anxiety disorder as set out by the DISC-P. To this end, we divided the full sample of clinical participants (i.e., ODD + $\mathrm{OD} / \mathrm{AD}+\mathrm{ADHD})$ in a subgroup with $(n=$ 30; ODD: $n=9$; OD/AD: $n=9$; and ADHD: $n=12)$ and without an anxiety disorder $(n=$ 36; ODD: $n=6$; OD/AD: $n=20$; and ADHD: $n=10)$. No significant differences between the subgroups with and without anxiety disorder were found in cortisol during the PRE and STRESS phase, nor did we find differences in cortisol between the subgroups with and without anxiety disorder within the separate externalizing groups (Mann-Whitney tests, with all $p$ values between .18 and .78).

\section{ANS: HR and skin conductance}

$H R$. There was no difference in HR between the 4 groups on entrance: $\mathrm{NC}=95.49(S D=$ $10.3), \mathrm{ODD}=86.1(S D=7.7), \mathrm{OD} / \mathrm{AD}=$ $92.1(S D=12.9), \mathrm{ADHD}=93.5(S D=13.6)$; $F(3,91)=2.11, n s$. A repeated-measures MANOVA indicated a main effect of time, $F$ $(8,84)=18.85, p<.001$, of group, $F(3$, $91)=2.68, p=.05$, and an interaction between group and time, $F(24,248)=3.24, p<.001$, with generally lower HR in the ODD group during the whole procedure (see Figure 2). Contrast tests indicated that the significant main effect of time was attributable to a general decrease between $\mathrm{hr} 1$ and hr2, $F=39.03$, $p<.001$, an increase between $\mathrm{hr} 2$ and $\mathrm{hr} 3$, $F=64.04, p<.001$, a decrease again between hr4 and hr5, $F=7.53, p<.007$, an increase between hr5 and hr6, $F=6.47, p<.02$, and again a general decrease between hr7 and hr8, $F=39.20, p<.001$, and between hr8 and hr9, $F=12.31, p<.002$. The significant interaction was mainly attributable to a decrease between hr5 and hr6 in the NC group, whereas the other three groups showed a HR increase at this point, $F=2.86, p<.05$, a decrease between hr6 and hr7 in the ADHD group, whereas this decrease was not present in the other groups, $F=3.34, p<.05$, a decrease between hr7 and hr8 in the ODD, OD/AD, and ADHD groups, but the NC group showed no such decrease, $F=6.78, p<.001$. This latter result is probably due to the fact that the NC children took part in an earlier study and were not informed about the outcome of the competition until the final sample, whereas the participants in the present study heard about the outcome just before sample 8. Post hoc Bonferroni tests showed significant differences between the NC and ODD group on hr5 $(p=.05)$, hr8 $(p=.001)$, and hr9 $(p<.02)$.

A repeated-measures MANOVA with $O D D$, $A D H D$, and $M P H$ as three separate between subjects factors showed that MPH, $F(1,89)=$ $4.69, p<.04$, but not the factors ODD, $F(1$, $89)=2.02, n s$, or ADHD, $F(1,89)=0.70, n s$, was responsible for the elevated HR levels found in the ADHD and OD/AD group. To further investigate the effect of MPH on HR, we compared children with ODD alone (who 


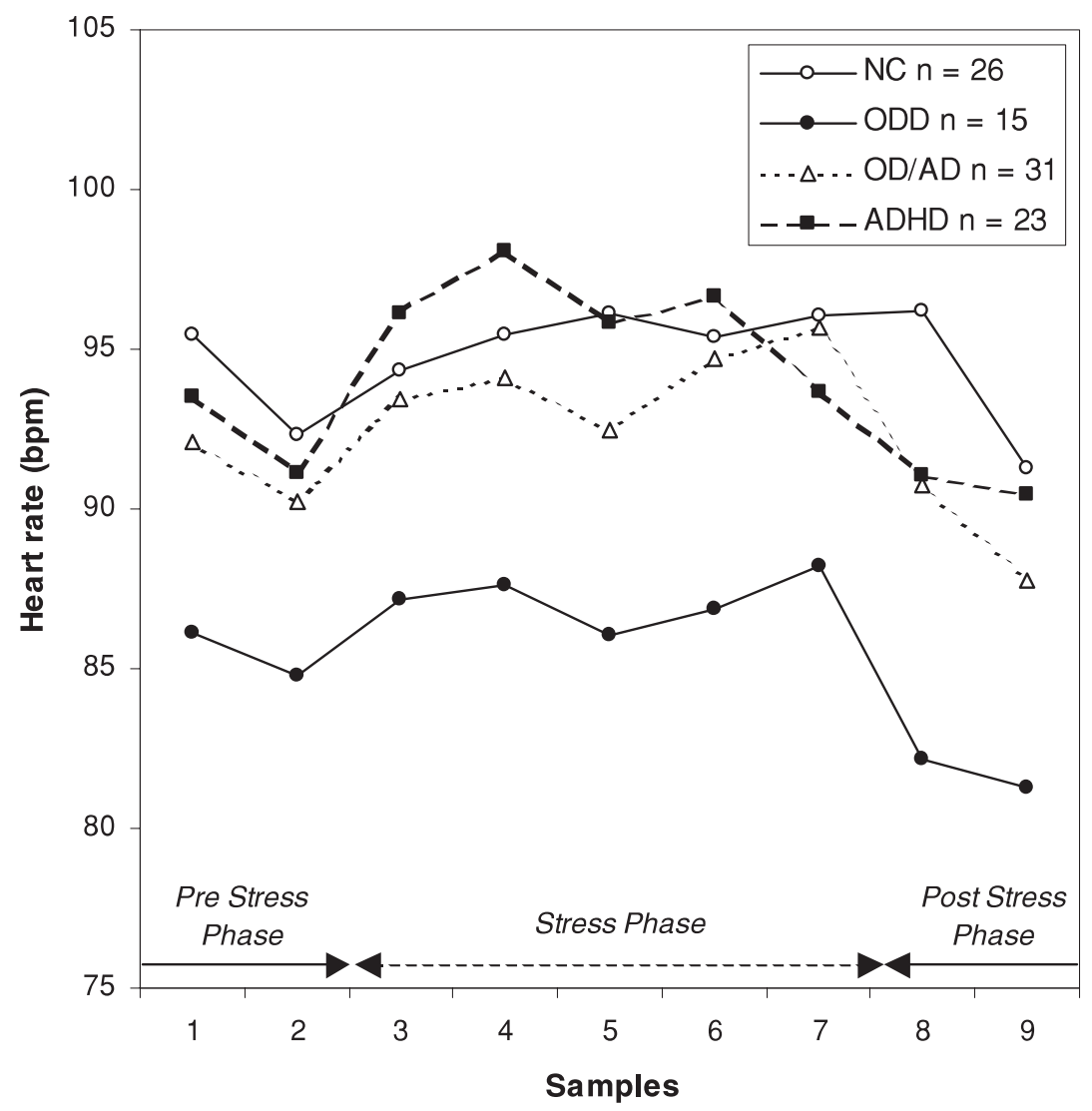

Figure 2. Mean heart rate levels in normal control (NC) children and children with oppositional defiant disorder (ODD), attention-deficit/hyperactivity disorder (ADHD), and ODD with comorbid ADHD $(\mathrm{OD} / \mathrm{AD})$.

are not on MPH; $n=15)$ to children with ADHD or $\mathrm{AD} / \mathrm{OD}$ who were not on $\mathrm{MPH}(n=15)$. No differences were found between these subgroups, suggesting that it is indeed MPH and not the factor ADHD that accounts for the elevated $H R$ in ADHD children using MPH: PRE phase $(=$ mean $\mathrm{hr} 1-\mathrm{hr} 2)$ : $\mathrm{ODD}=85.5(S D=$ 8.2), $\mathrm{ADHD}+\mathrm{AD} / \mathrm{OD}=86.6(S D=10.1), F$ $(1,28)=0.12, n s ;$ STRESS phase $(=$ mean $\mathrm{hr} 3-$ hr7): ODD $=87.2(S D=7.2),[$ ADHD + $\mathrm{AD} / \mathrm{OD}]=89.1(S D=10.5), F(1,28)=0.34$, $n s ;$ POST phase (=mean hr8-hr9): ODD $=81.8$ $(S D=7.2), \mathrm{ADHD}+\mathrm{AD} / \mathrm{OD}=85.4(S D=$ 9.6), $F(1,28)=1.42$, ns.

SCL analyses. Two subjects (1 ODD and 1 $\mathrm{OD} / \mathrm{AD}$ ) were excluded from the SCL analyses because they had four or more outlier values. The SCL data of one ODD participant were not available due to technical problems. Throughout the whole procedure NC children had higher SCL values compared to the three externalizing groups. (Post hoc Bonferroni tests showed $p$ values of all samples between .001 and .04.) Analyzing all 9 samples together, a main effect of time, $F(8,80)=74.26, p<$ .001 , and group, $F(3,87)=4.97, p<.004$, but no interaction between group and time, $F$ $(24,236)=1.45, n s$, were found (see Figure 3). Contrast tests indicated that the significant time effect was attributable to an increase in SCL from Sample 1 to Sample 7, after which the increase stopped. No effect of MPH on SCL could be established.

As in our previous study (Van Goozen, Matthys, et al., 2000), we found that changes in HR, SCL and cortisol as a result of the experimental procedure were not correlated in the 


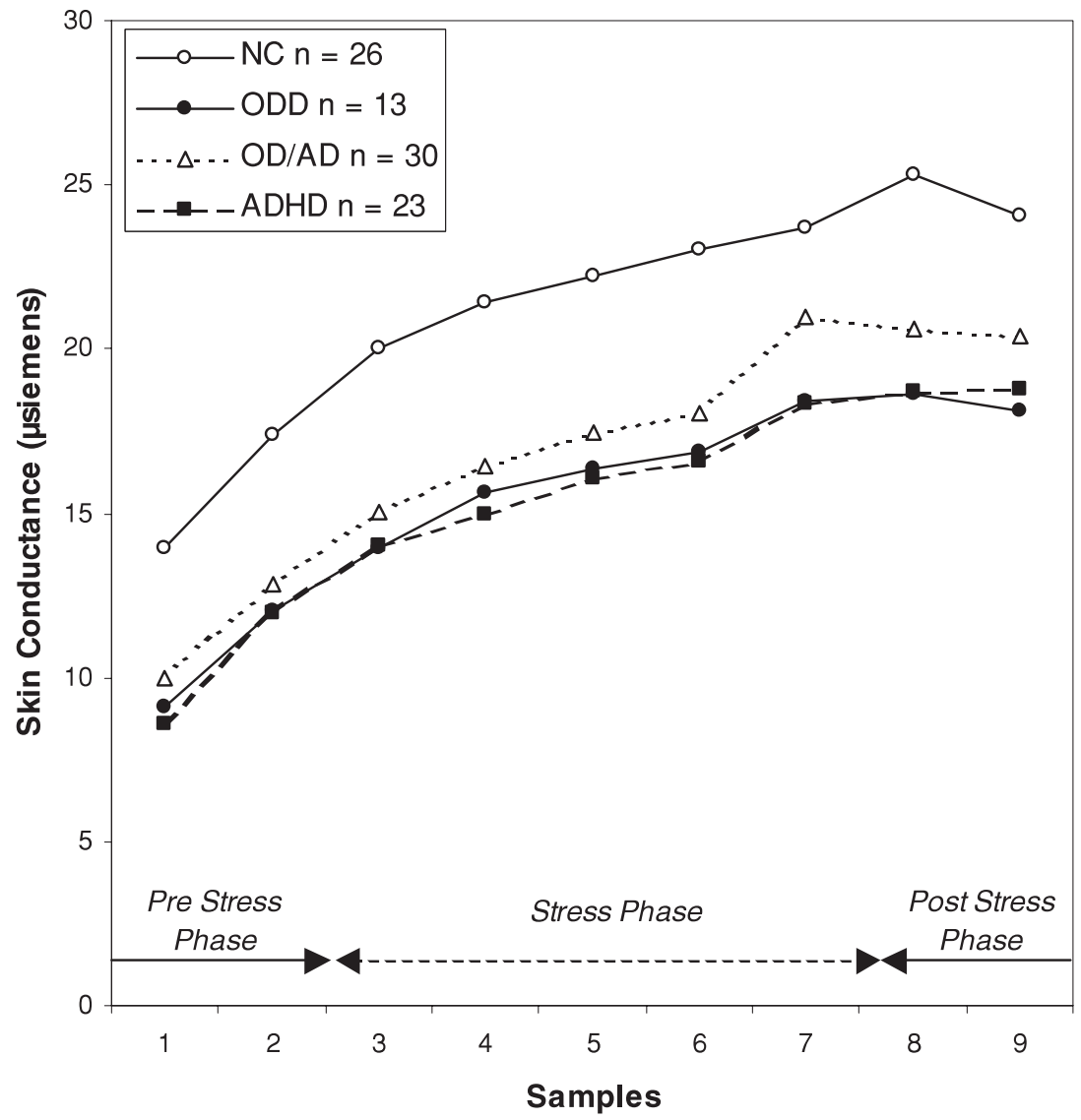

Figure 3. Mean skin conductance levels in normal control (NC) children and children with oppositional defiant disorder (ODD), attention-deficit/hyperactivity disorder (ADHD), and ODD with comorbid ADHD (OD/AD).

total sample. We only found significant positive correlations between the mean HR levels and mean cortisol levels during the pre stress phase $(r=.23, p=.03)$ and the stress phase $(r=.38, p<.001)$.

It has suggested that $\mathrm{CD}$ is a more serious condition than ODD. We, therefore, investigated whether there were differences between participants with ODD or CD, we combined the ODD and OD/AD groups and divided them in two subgroups; one consisting of children with ODD $(n=33)$ and one with children with $\mathrm{CD}(n=12)$. No differences were found in HR, SCL, and cortisol data between the ODD and CD subgroups.

Finally, no correlations were found between these biological measures and age, and no differences were found between boys and girls in HR, SCL, or cortisol.

\section{Emotional arousal}

Negative mood. The four groups were compared with respect to the self-reported intensity of their negative moods. Figure 4 clearly shows that on entrance the NC group reported significantly more intense negative moods, vz1: $F(3,91)=21.87, \mathrm{p}<.001$. A repeatedmeasures MANOVA revealed a main effect of time, $F(8,84)=14.90, p<.001$, of group, $F$ $(3,91)=10.55, p<.001$, and an interaction between group and time, $F(24,248)=4.92$, $p<.001$. Additional contrast tests showed that the main time effect was attributable to a general decline in negative mood between vz1 and $\mathrm{vz2}$, an increase between vz2 and vz3, and between vz3 and vz4, and again a decrease between vz5 and vz6. The significant interaction effect was mainly attributable to a 


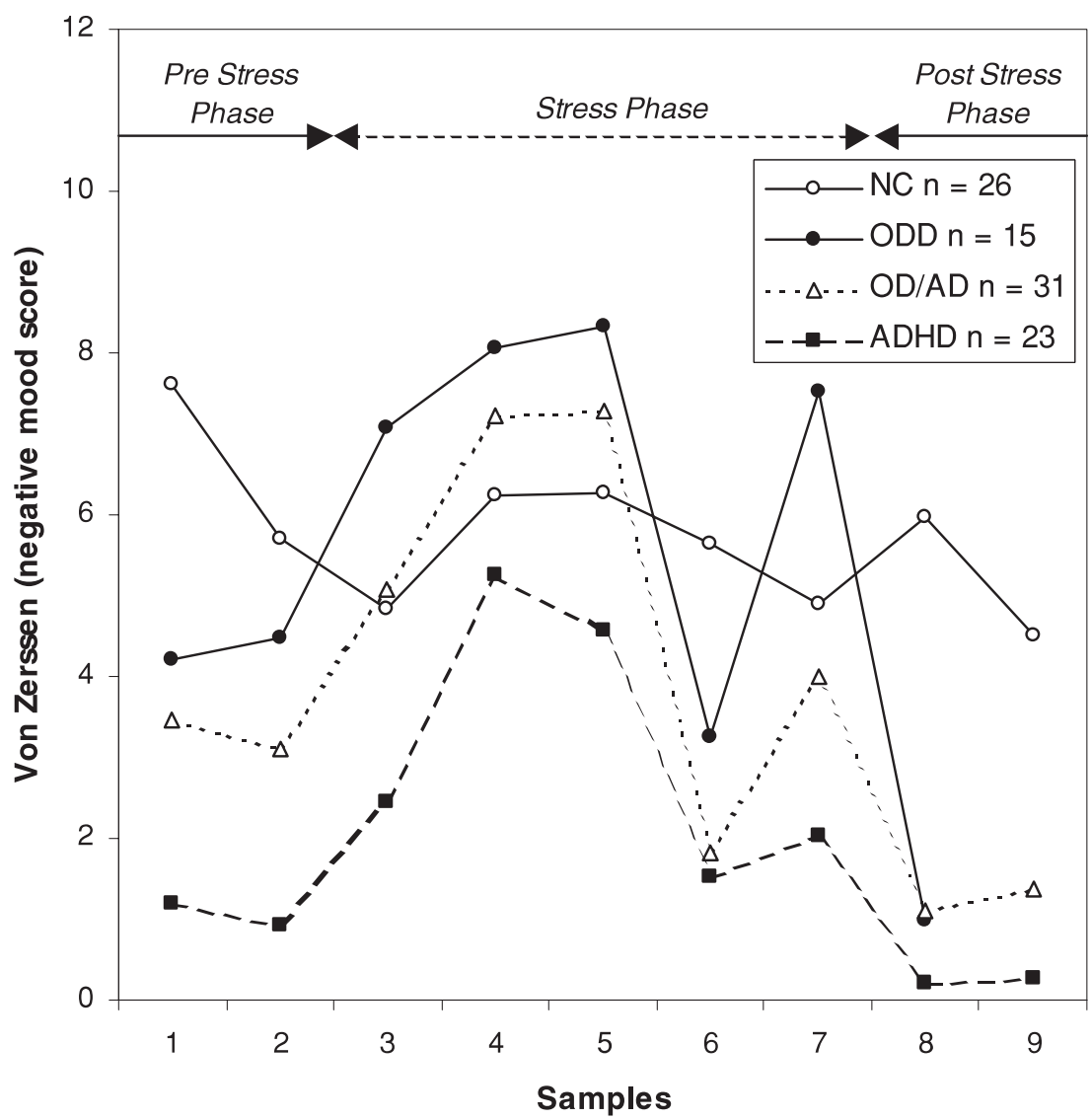

Figure 4. Mean negative mood scores in normal control (NC) children and children with oppositional defiant disorder (ODD), attention-deficit/hyperactivity disorder (ADHD), and ODD with comorbid $\mathrm{ADHD}(\mathrm{OD} / \mathrm{AD})$.

decrease in negative mood in the $\mathrm{NC}$ group between vz2 and vz3, whereas the three clinical groups reported an increase in negative mood between these samples. A similar interaction effect was found between vz6 and vz7. It seems to be the case that the clinical groups reported more intense negative mood after the frustrations tasks (block 3 and 7), whereas all four groups seemed to be equally affected by the frustration and provocation task in block 4 . The increase in negative mood between vz7 and vz8 in the NC group happened at a time the clinical groups reported a decrease. We already mentioned earlier that the NC children had to wait longer for a decision about the outcome of the competition than the clinical cases (see Table 2).

Finally, we found a significant difference between the four groups in the intensity of aggressive behavior towards their opponent, $F(3,91)=3.14, p<.03$, with both the ODD and the OD/AD group showing the highest intensity of aggression (mean aggression intensity in $\mathrm{ODD}=18.8$, in $\mathrm{OD} / \mathrm{AD}=18.3$, in $\mathrm{ADHD}=9.3$, and in $\mathrm{NC}=10.1$.

\section{Discussion}

Patterns of lower ANS and HPA axis activity have been found in children with externalizing behavior disorders (e.g., McBurnett et al., 2000; Van Goozen et al., 1998; Van Goozen, Matthys, et al., 2000). Thus far, the patient samples of studies investigating, for example, SCL, HR, and cortisol in children consisted of heterogeneous groups of children showing externalizing behavior: CD or ODD, but also children with ADHD, or ODD with comorbid 
Table 2. Design of the study

Experimental Phases

\begin{tabular}{|c|c|c|c|c|c|c|c|c|c|}
\hline & \multicolumn{2}{|c|}{ PRE Phase } & \multicolumn{5}{|c|}{ STRESS Phase } & \multicolumn{2}{|c|}{ POST Phase } \\
\hline & $0-15 \min$ & $16-30 \mathrm{~min}$ & $31-45 \mathrm{~min}$ & $46-65 \mathrm{~min}$ & $66-70 \mathrm{~min}$ & $71-95 \mathrm{~min}$ & $96-110 \mathrm{~min}$ & $111-125 \mathrm{~min}$ & $126-135 \mathrm{~min}$ \\
\hline Blocks & Block1 & Block2 & Block3 & Block4 & Block5 & Block6 & Block7 & Block8 & Block9 \\
\hline Tasks & Introduction & Video & Frustration & $\begin{array}{l}\text { Frustration }+ \\
\text { provocation }\end{array}$ & Provocation & Aggression & Frustration & $\begin{array}{l}\text { Video }+ \\
\quad \text { outcome }\end{array}$ & $\begin{array}{l}\text { Video }+ \\
\quad \text { outcome }^{a}\end{array}$ \\
\hline Cortisol $^{b}$ & & & Cort 1 & Cort2 & Cort3 & Cort4 & Cort5 & Cort6 & Cort7 \\
\hline SCL & Scl1 & $\mathrm{Scl} 2$ & $\mathrm{Scl} 3$ & $\mathrm{Scl} 4$ & Scl5 & Scl6 & $\mathrm{Scl} 7$ & Scl8 & Scl9 \\
\hline HR & Hr1 & $\mathrm{Hr} 2$ & $\mathrm{Hr} 3$ & $\mathrm{Hr} 4$ & Hr 5 & Hr6 & $\mathrm{Hr} 7$ & $\mathrm{Hr} 8$ & $\mathrm{Hr} 9$ \\
\hline $\begin{array}{l}\text { Negative } \\
\text { moods (VZ) }\end{array}$ & Vz1 & $\mathrm{Vz} 2$ & Vz3 & Vz4 & Vz5 & Vz6 & Vz7 & Vz8 & Vz9 \\
\hline
\end{tabular}

Note: HR, Heart rate; SCL, skin conductance Level; VZ, Von Zerssen Negative Mood scale; PRE, prestress; POST, poststress.

${ }^{a}$ The NC children who were included from a prior study were not informed about the outcome of the competition until the last sample vz9.

${ }^{b}$ Because of the delay in cortisol increase, samples cort 1 and cort 2 were considered the PRE phase samples and cort3-cort7 the STRESS phase samples. 
ADHD. The aim of this study was to investigate whether a pattern of lower ANS and HPA (re-) activity is present in externalizing behavior disorders in general or is only specifically found in children with ODD. To this end, we studied cortisol, HR, and SCL in 95 prepubertal children (26 NC children and 69 patients referred for externalizing behavior problems: 15 ODD, 31 OD/AD, and 23 ADHD) under baseline conditions but also during a psychological challenge, which involved the exposure to provocation and frustration and thereby induced anger.

No differences were found between the four groups in baseline levels of cortisol. These results are in contrast with the findings of lower baseline cortisol levels in CD children as reported by McBurnett et al. (2000). Like in earlier studies (Van Goozen et al., 1998; Van Goozen, Matthys, et al., 2000a), no clear cortisol response was found in both the ODD and $\mathrm{OD} / \mathrm{AD}$ groups when they were exposed to stress. These findings support our hypothesis that disturbances in HPA axis activity in ODD children only become evident during stress. This explains (or partly explains) why some studies did not find lower cortisol levels in aggressive children (Kruesi et al., 1989; Scarpa and Kolko, 1994; Schulz et al., 1997; Stoff et al., 1992). The NC and ADHD groups, on the other hand, responded to the psychological stressor with a clear increase in cortisol, and it is also relevant that the cortisol values of the ADHD children were very similar to those of the normal controls. These results indicate that a pattern of low HPA responsivity does not characterize the spectrum of externalizing behaviors as a whole, but is specifically present in children with ODD, whether or not they have comorbid ADHD. In future studies investigating HPA axis reactivity in children with externalizing behavior problems, it is therefore important to distinguish between disruptive children (i.e., those with ODD or CD), and children with ADHD alone.

It is interesting that the cortisol pattern of the OD/AD group was very similar to that of the ODD group, because it has been proposed that OD/AD children as a group are more seriously affected than children with ODD alone (Matthys, Van Goozen, de Vries, Cohen-
Kettenis, \& van Engeland, 1998). We did, however, find that the more severely affected (inpatient) ODD and OD/AD children had the lowest pattern of HPA axis activity during stress. Thus, severity of disruptive behavior disorder seems to be inversely related to the activity of the HPA axis under stress. Interestingly, and in a similar way as we found in our previous studies, ODD and OD/AD children did not report lower negative mood scores, and they behaved more aggressively than the ADHD and NC children. Thus, we observed in both ODD groups a discrepancy between the intensity of self-reported negative moods and intensity of aggression, on the one hand, and cortisol response, on the other. In the ADHD and NC children such a discrepancy was absent: they showed a correspondence between intensity of behavior, negative moods, and increase in HPA axis activity,

As for ANS activity, we observed that NC children had significantly higher SCL levels throughout the entire experimental procedure compared to the three externalizing groups, and no differences were found between the externalizing groups. Moreover, SCL levels were equally affected by stress in the four groups. With respect to HR, the ODD group had significantly lower HR levels during baseline and stressful conditions compared to the $\mathrm{NC}, \mathrm{OD} / \mathrm{AD}$, and ADHD groups. We were able to demonstrate that the higher HR levels in the ADHD and OD/AD groups were due to the MPH treatment of the participants in these subgroups.

The fact that the ODD children are not characterized by a general hypoactivity of the HPA axis but rather that their axis is particularly hyporesponsive during stress has some implications for the arousal theories. Specifically, the absence of lower baseline cortisol levels in ODD children is incompatible with the hypothesis that they might be driven to their behavior by stimulation-seeking motives. On the other hand, these findings seem to be supportive of the fearlessness theory, although it is unclear at the moment how these data should be explained. A possible explanation could be that very frequent exposures to stress have resulted in a habituation among ODD children to (some types of) stress, and that they there- 
fore show low stress responsivity. In addition, it does not seem to be the case that the apparent hyporesponsivity of the HPA axis in ODD children was dependent on their perception and interpretation of the stressor, because, as discussed previously, the ODD children clearly reported being negatively affected by what was happening to them. Furthermore, it has been shown that ODD children more readily interpret ambiguous situations in a hostile was (Dodge, 1993). Thus, according to our results, ODD children do indeed perceive stressors as threatening, frustrating or hostile, but somehow they do not show a cortisol increase. Third, it is possible that in ODD children their HPA axis and their subjective arousal are less well coordinated, perhaps by a permanent effect of stressful events in early life, on the developing neurobiological systems in the brain including the HPA axis. In contrast to the cortisol data, the low baseline SCL levels are compatible with the stimulation-seeking theory. The fact that ODD and ADHD children show lower SCL levels compared to NC children could mean that externalizing children in general are more prone to seek out stressful situations to increase their lower arousal (SCL) levels. Unfortunately, due to the treatment with MPH and the consequent elevation of HR, our HR data cannot provide further evidence in support of this hypothesis.

\section{Limitations}

Some limitations of the present study need to be addressed. The first limitation is the fact that nearly all ADHD subjects and about half of the OD/AD children were treated with MPH at the time of the assessment makes the HR findings difficult to interpret. The differences in HR between the ODD, OD/AD, and ADHD subgroups were clearly a result of the stimulating effect of MPH. Therefore, future studies should include ODD and ADHD groups without MPH to further investigate the difference in HR between these groups.

Kariyawasam et al. (2002) suggested that stimulant medication, such as MPH, could also influence cortisol levels. They found that lower cortisol was restricted to a subgroup of OD/AD children who did not receive stimu- lant medication compared to OD/AD patients who were prescribed MHP. We do not believe that treatment with MPH has influenced our cortisol data. First, we did not find a difference in baseline cortisol levels between NC, ADHD, OD/AD, and ODD children. Second, and even more important, we found no differences in cortisol responsivity between ODD children with and without MPH. It is possible that the OD/AD subgroup in the study by Kariyawasam et al. (2002) was diagnostically different from the one receiving MPH.

A second limitation is that we did not systematically collect information on the occurrence of significant early life events in our children nor examine the presence of a family history of psychiatric disorders. We can therefore only speculate about the possible mechanisms underlying our findings. Early adverse experiences, including neglect or abuse, can have permanent effects of the developing neurobiological systems in the brain, including the HPA axis (Carlson \& Earls, 1997). Two clinically based studies found that children with $\mathrm{CD}$ had been exposed to significantly greater environmental adversity than children with ADHD (Biederman, Munir, \& Knee, 1987; Schachar \& Tannock, 1995), and the HPA axis abnormalities that we found in the ODD and OD/AD children but not in the ADHD children could therefore have been caused by these differences in the early lives of the children we studied. Longitudinal prospective studies from an early age onward are needed in order to investigate the effect of adverse early life events on the HPA axis in a more detailed way. On the other hand, it goes without saying that differences in HPA axis functioning can also be due to genetic differences between families with disruptive children and normal children. A third limitation is that, although the present study shows that ODD and OD/AD children do not respond endocrinologically to challenges that involve frustration and provocation (and thereby induce anger), it is not clear to what extent these findings would generalize to other types of stress. Future research should examine how children with ODD respond, for example, to fear-inducing challenges. 
Finally, it should be acknowledged that the use of citric acid to stimulate saliva flow has the potential to cause artificially high results (Schwartz, Granger, Susman, Gunnar, \& Laird, 1998). Although at the time of the study it was still a relatively common practice to stimulate saliva production using citric acid, at present, the use of stimulants is not necessary because new generation assays for salivary biomarkers only require a small fraction of the volume once needed.

\section{Clinical implications}

The results of this study indicate that a pattern of low HPA responsivity during stress does not characterize the spectrum of externalizing behaviors as a whole, but is only specifically present in children with ODD, with or without comorbid ADHD. The finding that ODD and ADHD children can be distinguished on the basis of the reactivity of their HPA axis supports the distinction between ODD and ADHD based on the behavioral criteria as set out in the DSM-IV (APA, 1994). Furthermore, HPA axis reactivity might be related to social conditioning as occurring in everyday child rearing. The better prognosis of ADHD than ODD (with or without ADHD), then, could be the result of a better responsivity to social conditioning due to a normal reactivity of the HPA axis. Moreover, it is also relevant from a clinical point of view that we found that the pattern of low HPA axis activity under stress was more apparent in more severely disturbed ODD children. At the moment, medication has only a small role to play in the treatment of ODD. In the future, it is possible that pharmacological interventions, which influence the activity of the HPA axis, become an interesting treatment option for children with persistent ODD. A better understanding of the mechanisms involved in the development, persistence and prognosis of disruptive behavior, including a better knowledge of the biological predispositions to antisocial behavior, should ultimately result in earlier and more effective interventions.

\section{References}

Abikoff, H., \& Klein, R. G. (1992). Attention-deficit hyperactivity and conduct disorder. Comorbidity and implications for treatment. Journal of Consulting and Clinical Psychology, 60, 881-892.

Achenbach, T. M. (1991). Manual for the Child Behavior Checklist and Profile. Burlington, VT: University of Vermont, Department of Psychiatry.

American Psychiatric Association. (1994). Diagnostic and statistical manual of mental disorders (4th ed.). Washington, DC: Author.

Biederman, J., Munir, K., \& Knee, D. (1987). Conduct and oppositional disorder in clinically referred children with attention deficit disorder: A controlled family study. Journal of the American Academy of Child and Adolescent Psychiatry, 26, 724-727.

Carlson, M., \& Earls, F. (1997). Psychological and neuroendocrinological sequelae of early social deprivation in institutionalized children in Romania. Annals of the New York Academy of Sciences, 807, 419-428.

Daugherty, T. K., \& Quay, H. C. (1991). Response perseveration and delayed responding in childhood behavior disorders. Journal of Child Psychology and Psychiatry, 32, 453-461.

Dodge, K. A. (1993). Social-cognitive mechanisms in the development of conduct disorder and depression. Annual Review of Psychology, 44, 559-584.

Fisher, P., Wicks, B. A., Shaffer, D., Piacentini, J., \& Lapkin, J. (1992). Diagnostic Interview Schedule for Children (DISC 2.3) parent version. New York: Columbia University Press (Translated into Dutch by F. C. Verhulst \& M. Kasius).

Fowles, D. C., \& Furuseth, A. M. (1994). Electrodermal hyporeactivity and antisocial behavior. In D. K. Routh (Ed.), Disruptive behavior disorders in childhood (pp. 181-205). New York: Plenum Press.

Frick, P. J., \& Loney, B. R. (1999). Outcomes of children and adolescents with oppositional defiant disorder and conduct disorder. In H.C. Quay \& A.E. Hogan (Eds.), Handbook of disruptive disorders (pp. 507-524). New York: Kluwer Academic/Plenum Press.

Gerra, G., Zaimovic, A., Avanzini, P, Chittolini, B., Giucastro, G., Caccavari, R., Palladino, M., Maestri, D., Monica, C., Delsignore, R., \& Brambilla, F. (1997). Neurotransmitter-neuroendocrine responses to experimentally induced aggression in humans: influence of personality variable. Psychiatry Research, 66, 33-43.

Halperin, J. M., Vanshdeep, S., Siever, L. J., Schwartz, S. T., Matier, K., Wornell, G., \& Newcorn, J. H. (1994). Serotonergic function in aggressive and nonaggressive boys with attention deficit hyperactivity disorder. The American Journal of Psychiatry, 151, 243-248.

Herpertz, S. C., Wenning, B., Mueller, B., Qunaibi, M., Sass, H. \& Herpertz-Dahlmann, B. (2001). Psychophysiological responses in ADHD boys with and without conduct disorder: Implications for adult antisocial behavior. Journal of the American Academy of Child and Adolescent Psychiatry, 40, 1222-1230.

Hinshaw, S. P. (1992). Externalizing behavior problems and academic underachievement in childhood and adolescence: Causal relationships and underlying mechanisms. Psychological Bulletin, 111, 127-155. 
Iaboni, F., Douglas, V. I., \& Ditto, B. (1997). Psychophysiological response of ADHD children to reward and extinction. Psychophysiology, 34, 116-123.

Kariyawasam, S. H., Zaw, F., \& Handley, S. L. (2002). Reduced salivary cortisol in children with comorbid attention deficit hyperactivity disorder and oppositional defiant disorder. Neuroendocrinology Letters, $23,45-48$.

Kirschbaum, C., \& Hellhammer, D. H. (1989). Salivary cortisol in psychobiological research: An overview. Neuropsychobiology, 22, 150-169.

Kirschbaum, C., Steyer, R., Eid, M., Patalla, U., Schwenkmezger, P. \& Hellhammer, D. H. (1990). Cortisol and behavior: 2. Application of a latent state-trait model to salivary cortisol. Psychoneuroendocrinology, 15, 297-307.

Klimes-Dougan, B., Hastings P. D., Granger, D. A., Usher, B. A., \& Zahn-Waxler, C. (2001). Adrenocortical activity in at-risk and normally developing adolescents: individual differences in salivary cortisol basal levels, diurnal variation, and responses to social challenges. Development and Psychopathology, 13, 695-719.

Kruesi, M. J. P., Hibbs, E. D., Zahn, T. P., Keysor, C. S., Hamburger, S. D., Bartko, J. J., \& Rapoport, J. L. (1992). A 2-year prospective follow-up study of children and adolescents with disruptive behavior disorders. Archives of General Psychiatry, 49, 429-435.

Kruesi, J. P., Schmidt, M. E., Donnelly, M., Hibbs, E. D., \& Hamburger, S. D. (1989). Urinary free cortisol output and disruptive behavior in children. Journal of the American Academy of Child and Adolescent Psychiatry, 28, 441-443.

Lahey, B. B., Loeber, R., Quay, H., Frick, P., \& Grimm, J. (1992). Oppositional-defiant and conduct disorders: Issues to be resolved in DSM-IV. Journal of the American Academy of Child and Adolescent Psychiatry, 31, 539-546.

Lahey, B. B., McBurnett, K., Loeber, R., \& Hart, E. L. (1995). Psychobiology. In G. Pirooz Sholevar (Ed.), Conduct disorders in children and adolescents (pp. 27-44). Washington, DC: American Psychiatric Press.

Loeber, R., \& Stouthamer-Loeber, M. (1998). Development of juvenile aggression and violence. Some common misconceptions and controversies. The American Psychologist, 53, 242-259.

Mannuzza, S., \& Klein, R. G. (1999). Adolescent and adult outcomes in attention-deficit/hyperactivity disorder. In H. C. Quay \& A. E. Hogan (Eds.), Handbook of disruptive disorders (pp. 297-293). New York: Kluwer Academic/Plenum Press.

Matthys, W., van Goozen, S. H. M., de Vries, H., CohenKettenis, P. T., \& van Engeland, H. (1998). The dominance of behavioural activation over behavioural inhibition in conduct disordered boys with and without attention deficit hyperactivity disorder. Journal of Child Psychology and Psychiatry, 39, 643-651.

McBurnett, K., Lahey, B. B., Frick, P. J., Risch, C., Loeber, R., Hart, E. L., Christ, M. A. G., \& Hanson, K. S. (1991). Anxiety, inhibition, and conduct disorder in children: II. Relation to salivary cortisol. Journal of the American Academy of Child and Adolescent Psychiatry 30, 192-196.

McBurnett, K., Lahey, B. B., Rathouz, P. J., \& Loeber, R. (2000). Low salivary cortisol and persistent aggression in boys referred for disruptive behavior. Archives of General Psychiatry, 57, 38-43.
Moss, H. B., Vanyukov, M. M., \& Martin, C. S. (1995). Salivary cortisol responses and the risk for substance abuse in prepubertal boys. Biological Psychiatry, 38, 547-555.

Pajer, K., Gardner, W., Rubin, R. T., Perel, J., \& Neal, S. (2001). Decreased cortisol levels in adolescent girls with conduct disorder. Archives of General Psychiatry, 58, 297-302.

Pennington, B. F., \& Ozonoff, S. (1996). Executive functions and developmental psychopathology. Journal of Child Psychology and Psychiatry, and Allied Disciplines, 37, 51-87.

Raine, A. (1993). The psychopathology of crime: Criminal behavior as a clinical disorder. San Diego: Academic Press.

Raine, A. (1996). Autonomic nervous system activity and violence. In D. M. Stoff \& R. B. Cairns (Eds.), Aggression and violence (pp. 145-168). Mahwah, NJ: Erlbaum.

Raine, A., \& Venables, P. H. (1984). Electrodermal nonresponding, antisocial behavior, and schizoid tendencies in adolescents. Psychophysiology, 21, 424-433.

Raine, A., Venables, P. H., \& Mednick, S. A. (1997). Low resting heart rate at age 3 years predisposes to aggression at age 11 years: Evidence from the Mauritius Child Health Project. Journal of the American Academy of Child and Adolescent Psychiatry, 36, 1457-1464.

Raine, A., Venables, P. H., \& Williams, M. (1990). Relationships between central and autonomic measures of arousal at age 15 years and criminality at age 24 years. Archives of General Psychiatry, 47, 1003-1007.

Scarpa, A., Fikretoglu, D., \& Luscher, K. (2000). Community violence exposure in a young adults sample: II. Psychophysiology and aggressive behavior. Journal of Community Psychology, 28, 417-425.

Scarpa, A., \& Kolko, D. J. (1994). Salivary testosterone and cortisol in disruptive children: Relationship to aggressive, hyperactive, and internalizing behaviors. Journal of the American Academy of Child and Adolescent Psychiatry, 33, 1174-1184.

Schachar, R. (1991). Childhood hyperactivity. Journal of Child Psychology and Psychiatry, and Allied Disciplines, 32, 155-191.

Schachar, R., \& Logan, G. (1990). Impulsivity and inhibitory control in normal development and childhood psychopathology. Developmental Psychology, 26, 710-720.

Schachar, R., \& Tannock, R. (1995). Test of four hypotheses for the comorbidity of attention-deficit hyperactivity disorders and conduct disorder. Journal of the American Academy of Child and Adolescent Psychiatry, 34, 639-648.

Schulz, K. P., Halperin, J. M., Newcorn, J. H., Sharma, V., \& Gabriel, S. (1997). Plasma cortisol and aggression in boys with ADHD. Journal of the American Academy of Child and Adolescent Psychiatry, 36, 605-609.

Schwartz, E. B., Granger D. A., Susman, E. J., Gunnar, M. R., \& Laird, B. (1998). Assessing salivary cortisol in studies of child development. Child Development, 69, 1503-1513.

Stoff, D. M., Pasatiempo, A. P., Yeung, J., Cooper, T. B., Bridger, W. H., \& Rabinovich, H. (1992). Neuroendocrine responses to challenge with $d l$-fenfluramine and aggression in disruptive behavior disorders of children and adolescents. Psychiatry Research, 43, 263-276. 
Tennes, K., \& Kreye, M. (1985). Children's adrenocortical responses to classroom activities and tests in elementary school. Psychosomatic Medicine, 47, 451-460.

Tennes, K., Kreye, M., Avitable, N., \& Wells, R. (1986) Behavioral correlates of excreted catecholamines and cortisol in second-grade children. Journal of the American Academy of Child and Adolescent Psychiatry, 25, 764-770.

Van Goozen, S. H. M., Matthys, W., Cohen-Kettenis, P. T., Buitelaar, J. K., \& Van Engeland, H. (2000). Hypothalamic-pituitary-adrenal axis and autonomic nervous system activity in disruptive children and matched controls. Journal of the American Academy of Child and Adolescent Psychiatry, 39, 1438-1445.

Van Goozen, S. H. M., Matthys, W., Cohen-Kettenis, P. T., Gispen-de Wied, C., Wiegant, V. M., \& Van Engeland, H. (1998). Salivary cortisol and cardiovascular activity during stress in oppositional defiant disorder boys and normal controls. Biological Psychiatry, 43, 531-539.

Van Goozen, S. H. M., Van den Ban, E., Matthys, W., Cohen-Kettenis, P. T., Thijssen, J. H. H., \& Van Engeland, H. (2000). Increased adrenal androgen functioning in children with oppositional defiant disorder: A comparison with psychiatric and normal controls. Journal of the American Academy of Child and Adolescent Psychiatry, 39, 1446-1451.
Vanyukov, M. M., Moss, H. B., Plail, J. A., Blackson, T., Mezzich, A. C., \& Tarter, R. E. (1993). Antisocial symptoms in preadolescent boys and in their parents: Associations with cortisol. Psychiatry Research, 46, 9-17.

Virkkunen, M. (1985). Urinary free cortisol secretion in habitually violent offenders. Acta Psychiatrica Scandinavica, 72, 40-44.

Von Zerssen, D. (1986). Clinical self-rating scales (CSRS of the Munich psychiatric information system). In N. Sartorius \& T. A. Ban (Eds.), Assessment of depression (pp. 270-303). Berlin: Springer-Verlag.

Weizman, R., Dick, J., Gil-Ad, I., Weitz, R., Tyano, S., \& Laron, Z. (1987). Effects of acute and chronic methylphenidate administration on $\beta$-endorphin, growth hormone, prolactin and cortisol in children with attention deficit disorder and hyperactivity. Life Sciences, 40, 2247-2252.

Woodman, D. D., Hinton, J. W., \& O’Neill, M. T. (1978). Cortisol secretion and stress in maximum security hospital patients. Journal of Psychosomatic Research, 22, 133-136.

Zahn, T. P., \& Kruesi, M. J. P. (1993). Autonomic activity in boys with disruptive behavior disorders. Psychophysiology, 30, 605-614.

Zuckerman, M. (1979). Sensation seeking: Beyond the optimal level of arousal. Hillsdale, NJ: Erlbaum. 